\title{
A Distinct Region in Erythropoietin that Induces Immuno/Inflammatory Modulation and Tissue Protection
}

\author{
RuiRong Yuan ${ }^{1,2}$ • Bo Wang ${ }^{1,2}$ - Wei Lu ${ }^{1}$ Yasuhiro Maeda ${ }^{1,2}$ Peter Dowling ${ }^{1,2}$
}

Published online: 14 August 2015

(C) The American Society for Experimental NeuroTherapeutics, Inc. (outside the U.S.) 2015

\begin{abstract}
Beneficial effects of short-term whole-molecule erythropoietin (EPO) therapy have been demonstrated on several animal models of diverse central nervous system pathology. However, the increased hematocrit induced by EPOdriven marrow stimulation greatly limits its potential for side effect-free therapy. We created a library of EPO-derived fragments based on the hypothesis that 2 distinct functions, erythropoiesis and tissue protection, reside in different regions of the molecule. Several small EPO-derived peptides within the A $\beta$ loop of whole EPO molecule were screened for tissue protection in EAE mice. The 19-mer JM-4 peptide that contains 2 cysteine molecules consistently demonstrated the most potent clinical beneficial effects without producing hematocrit alterations in animal models of EAE. The JM-4-induced tissue protection was associated with modulation of the immunoregulatory process that drives inflammation and provokes subsequent autoimmune damage. Like the whole EPO molecule, JM-4 effectively modulated immune/inflammatory reaction within both the peripheral lymphatic tissue and central nervous system. The major effects induced by JM-4 include blocked expansion of monocyte/dendritic antigen presenting cell and $\mathrm{T}$ helper 17 cell populations, decreased proinflammatory cytokine production, and sharply enhanced expansion of the regulatory T-cell population. JM-4 shows promise for treatment of a broad spectrum of neural and non-neural conditions associated with inflammation.
\end{abstract}

Peter Dowling

dowlinpc@njms.rutgers.edu

1 Neurology Service, VA Medical Center of East Orange, East Orange, NJ, USA

2 Department of Neurology and Neurosciences, Rutgers New Jersey Medical School, Newark, NJ, USA
Keywords Multiple sclerosis · Experimental autoimmune encephalomyelitis · Immunomodulation · Erythropoietin · Peptide

\section{Introduction}

Erythropoietin (EPO), a 165-amino acid glycoprotein cytokine, is traditionally known to play an important role as a renal hormone that regulates hematopoiesis by binding and stimulating erythroid progenitor cell proliferation in the bone marrow. In addition, many studies have shown that EPO also possesses other diverse biological functions, including antiinflammatory, antiapoptotic, and tissue protective effects [1-5]. Exogenously administered EPO is protective in several animal models of neurologic injury, including experimental autoimmune encephalomyelitis (EAE), traumatic brain injury, and stroke [6-11]. Our group has previously demonstrated that whole-molecule EPO has marked beneficial immunomodulatory and tissue protective effects, and appears to target specific immune cells critical to the induction and maintenance of EAE [12]. However, 1 major obstacle in translating these findings into a therapeutic intervention for human disease is that EPO significantly elevates red cell mass, which can lead to hypertension, stroke, and other serious cardiovascular complications [13-15].

EAE is an animal model of multiple sclerosis that possesses similar pathologic hallmarks, including mononuclear inflammatory infiltration in the central nervous system (CNS) white matter with subsequent demyelination and axonal dysfunction [16-19]. Described as a T-cell-mediated disease, EAE is induced by immunization with neural antigens, such as myelin oligodendrocyte glycoprotein (MOG) or proteolipid protein (PLP), that are subsequently presented by dendritic cells (DCs) to $\mathrm{T}$ cells within the lymphoid tissue and over the 
leptomeningial surface of the brain and spinal cord [17]. Activated $\mathrm{T}$ cells then initiate a pro-inflammatory cytokine cascade that ultimately disrupts tolerance to self-antigen and provokes an immune attack against host CNS white matter [20-24]. In a previous study, we found that the wholemolecule form of EPO blocks proliferation in MOG-reactive $\mathrm{T}$ cells, dendritic and mononuclear cells (MNC), suppresses cytokine production, and expands the regulatory $\mathrm{T}$ cell (Treg) population in lymphoid and CNS target tissue [12].

We hypothesized that the erythropoietic and tissueprotective elements of EPO reside in different domains of the molecule and the domains for tissue protection could be dissected from the regions responsible for erythropoiesis within the whole EPO molecule. We generated a set of EPOderived small peptides that contain either 1 or 2 cysteine(s) within a 7- to 25-mer peptide based on this hypothesis. After screening them for biologic activity and stability utilizing different in vitro culture systems, we then used 2 animal models of EAE to ascertain whether the small EPO peptides would retain immunomodulatory properties in EAE animals. We identified one 19-mer JM-4 peptide that contains a disulfide bond motif from the EPO-derived peptide library and demonstrated that it was highly protective when administered to symptomatic EAE mice immunized with either PLP or MOG antigen. It is notable that the strong tissue protective effects were achieved without significant hematologic/ immunologic side effects.

\section{Materials and Methods}

\section{EPO and Peptides}

EPO (Epoetin Alfa, Ortho Biotech, Titusville, NJ, USA) in 2000 $\mathrm{U} / \mathrm{ml}$ vial stock was stored at $4^{\circ} \mathrm{C}$ for in vitro or in vivo experiments. PLP ${ }_{139-151}$ peptide (HSLGKWLGHPDKF) and $\mathrm{MOG}_{35-}$ ${ }_{55}$ (MEVGWYRSPFSRVVHLYRNGK) peptides, EPO-derived small peptides (17-mer: AEHCSLNENITVPDTKV; JM-4: GCAEHCSLNENITVPDTKV), or control peptide $\mathrm{P} 60_{217-225}$ (KYGVSVQDI) were synthesized and purified by United Biochemical Research (Seattle, WA, USA). Peptides were synthesized using solid phase techniques and purified by highperformance liquid chromatography with $90 \%$ purity. Peptides were dissolved in phosphate-buffered saline (PBS; at $1 \mathrm{mg} / \mathrm{ml}$ ) and kept at $-20^{\circ} \mathrm{C}$ before use.

\section{Mice}

SJL/J (6-8 weeks old) and C57BL/6 (8-10 weeks old) female mice were purchased from Charles River Laboratories (Wilmington, MA, USA) and maintained in a conventional facility receiving standard diet and water ad libitum. The studies were conducted in accordance with the Animal
Component of Research Protocol guidelines at the VA Medical Center, East Orange, NJ, USA and with the United States Public Health Service's Policy on Humane Care and Use of Laboratory Animals.

\section{Animal Model of EAE and Clinical Evaluation}

The effect of our small EPO peptides and whole EPO molecule were assayed on the clinical course in the $\mathrm{SJL} / \mathrm{J}$ and C57BL/6 mouse models of EAE [7, 12, 16-18, 25]. Briefly, SJL mice were immunized subcutaneously at the tail base with $100 \mu \mathrm{l}$ of peptide emulsion containing $150 \mu \mathrm{g}$ PLP $_{139-151}$ antigen and an equal volume of complete Freund's adjuvant supplemented with $200 \mu \mathrm{g}$ Mycobacterium tuberculosis H37Ra. C57BL/6 mice were immunized subcutaneously at the tail base with $150 \mu \mathrm{g}$ MOG peptide ${ }_{35-55}$ dissolved in distilled water and emulsified with an equal volume of complete Freund's adjuvant supplemented with $4 \mathrm{mg} / \mathrm{ml}$ M. tuberculosis H37Ra. Immediately after immunization, animals received an intravenous injection of $200 \mathrm{ng}$ Bordetella pertussis toxin (List Biological Laboratories, Campbell, CA, USA) in $200 \mu \mathrm{l}$ PBS. To determine if JM-4 could prevent disease exacerbation, SJL/J mice were first immunized with a suboptimal dose of PLP peptide $(100 \mu \mathrm{g})$ on day 0 and subsequently challenged with the same amount of PLP antigen after a 7-day course of treatment with either JM-4 or sham treatment with PBS. Animals were weighed daily and assessed for clinical signs of EAE by 2 independent blinded observers as previously described [7].

\section{Therapy with Full-length EPO or Short EPO-derived Peptides}

Treatment with whole EPO or EPO-derived short peptides was initiated 24-48 h after the onset of neurologic signs (average clinical score 3-5). Groups of clinically symptomatic SJL/J EAE animals (6-10 per group) were treated intravenously with either $250 \mu \mathrm{g} / \mathrm{kg} /$ day $(\sim 5 \mu \mathrm{g} /$ mouse) EPO peptides or PBS for the same treatment period. A third group of clinically symptomatic C57BL/6 EAE animals was treated with intravenous full-length EPO (500 U/kg/day) for the same treatment period.

\section{Hematocrit}

Groups of 5 normal SJL or C57 black female mice aged 6-8 weeks were given daily intravenous full-length EPO (50-500 $\mathrm{U} / \mathrm{kg}$ ), short EPO peptides $200-500 \mu \mathrm{g} / \mathrm{kg}$ in PBS, or PBS alone for 7-14 days. Blood was collected weekly for 5 weeks to document changes in red blood cell mass, as measured by alterations in hematocrit. 


\section{Tissue Histology Correlation}

Nervous system tissue preparation for routine histology, detection of blood-brain barrier breakdown, and immunohistochemical examinations were performed as previously described [7]. Briefly, animals were perfused with 4\% paraformaldehyde in $0.1 \mathrm{M}$ phosphate buffer and their brains and spinal cords were removed and placed in 4\% paraformaldehyde and then embedded in paraffin. Luxol fast blue/periodic acid-Schiff stain was used for tissue examination. Fivemicrometer-thick sections were cut from brain and spinal cord regions. Each slide was constructed to contain normal control tissue sections, sham-treated EAE sections, and JM-4 treated EAE sections. For SMI-32 staining, 5- $\mu$ m-thick sections were deparaffinized, treated with antigen-retrieving solution, and blocked to minimize nonspecific binding. The sections then were incubated with mouse SMI-32 antibody (Sternberger Mono-clonals, Lutherville, MD, USA) at 1 to 5000 dilution in PBS overnight at $4^{\circ} \mathrm{C}$. After 2 PBS washes, the sections were incubated with biotinylated horse antimouse IgG (Vector Labs, Burlingame, CA, USA), followed by horseradish peroxidase-avidin complex (Vector Labs) for $30 \mathrm{~min}$ each. Immunoreactivity was visualized by exposing sections to diaminobenzidine tetrahydrochloride (Stable DAB; Invitrogen, Carlsbad, CA, USA) for 1-3 min. Excess DAB was removed by washing with distilled water, and then the slides were coverslipped and evaluated by light microscopy.

\section{Peripheral Inflammatory Cells, Flow Cytometry and Enriched EAE T-cell Cytokine Levels}

The total MNCs were quantified from draining inguinal lymph nodes (DILNs) of C57 EAE mice after treatment for 3-10 days with whole EPO or JM-4, or PBS. Cell surface staining of mouse single cell suspensions from C57 EAE DILNs was obtained with monoclonal antibodies directed against CD3, CD4, and CD11c (BD PharMingen, San Diego, CA, USA). For intracellular interleukin (IL)-17 or Foxp3 staining, MNCs were obtained either from DILNs or spleens of EAE mice after 5 days of treatment with PBS or JM-4. Cells were first reacted with fluorescein isothiocyanateanti-CD4 (eBioscience, San Diego, CA, USA), then washed, fixed, and permeabilized following the manufacturer's recommendations. The phycoerythrin-conjugated anti-Foxp3 or anti-IL-17 monoclonal antibody was subsequently added [12]. Flow cytometry was performed on a BD FACScan machine (BD Biosciences, San Jose, CA, USA) and data were analyzed using CellQuest software (BD Biosciences) and the FLOWJO (FLOWJO, Portland, OR, USA) analysis program.

T cells were collected and purified from spleen and DILNs of C57BL/6 EAE mice 5-7 days after the second immunization with $\mathrm{MOG}_{35-55}$, and the cells were cultured with synergic spleen antigen presenting cells pulsed with $\mathrm{MOG}_{35-55}$ peptide
[12]. Luminex technology was used to quantify multiple cytokines within the enriched EAE T-cell culture supernatants 72-96 h after incubation. Commercial kits (Upstate Biotechnology, Lake Placid, NY, USA) containing multicytokine beads (IL-2, IL-5, IL-6, tumor necrosis factor- $\alpha$ and interferon- $\gamma$ ) were utilized following the manufacturer's instructions [7].

\section{A $\beta$ Cytotoxicity Assay}

The rat PC12 pheochromocytoma cell line (ATCC, Manassas, VA, USA) was cultured in suspension and seeded onto 12well poly-D-lysine coated plates at a concentration of 3-5 $\times$ $10^{5}$ cells $/ \mathrm{ml}$ in Dulbecco's Modified Eagle Medium with $10 \%$ horse serum plus 5\% fetal bovine serum and cultured for $24 \mathrm{~h}$. The cells were differentiated further with Dulbecco's Modified Eagle Medium containing 1\% horse serum plus $100 \mathrm{ng} / \mathrm{ml}$ nerve growth factor for another $24 \mathrm{~h}$. Cultures were pretreated with JM-4 or scrambled JM-4 at a concentration of $1 \mu \mathrm{g} / \mathrm{ml}$ for $2 \mathrm{~h}$, and then challenged with $\mathrm{A} \beta_{25-35}$ peptide at $40 \mu \mathrm{M}$ for $24 \mathrm{~h}$. The cells were harvested and analyzed for death using allophycocyanin conjugated-annexin $\mathrm{V}$ and 7amino-actinomycin D (7-AAD) staining following the manufacturer's protocol (BD Biosciences). Washed cells were analyzed by flow cytometry within $30 \mathrm{~min}$ of the reaction.

\section{A $\beta$ Exposure and PC12 Apoptosis Assay Analyzing Cytoprotection by Various Segments of EPO}

Rat PC12 cell line was cultured and differentiated as above. Cells were then pretreated with JM-4, scrambled JM-4, EPO, or 1 of 6 peptide segments derived from EPO (labeled EPP\#1-EP-P\#6 with each segment spanning 25 amino acids of the primary structure of EPO beginning at the $\mathrm{N}$ terminus, except for EP-P\#6, which spans amino acids D136-166R) at a concentration of $1 \mu \mathrm{g} / \mathrm{ml} 2 \mathrm{~h}$ prior to adding $\mathrm{A} \beta_{25-35}$ peptide at $40 \mu \mathrm{M}$. Twenty-four hours after addition of $\mathrm{A} \beta_{25-35}$ peptide, cells were harvested and analyzed by fluorescenceactivated cell sorting using allophycocyanin conjugated Annexin $\mathrm{V}$ binding and 7-amino-actinomycin D (7-AAD) staining.

\section{Statistical Analysis}

Data are presented as the mean \pm SEM. The composite data were analyzed by the Kruskal-Wallis 1-way analysis of variance. The Mann-Whitney $U$ test was used to determine the significance of the intergroup difference in EAE clinical scores and duration of maximum neurological deficit. Values of $p<0.05$ were considered significant. Cytotoxicity assays were performed in duplicate and repeated independently 3 times. 


\section{Results}

\section{Short EPO-derived Peptides do not Elevate Hematocrit}

We previously reported that full-length EPO has marked beneficial effects on the acute neurological deficit in EAE C57BL/6 mice without significant hematocrit alteration, even with daily high-dose EPO (500-1000 U/kg/day for 7-14 days). However, while initial clinical improvement was observed when the same EAE protocol was conducted in SJL/J mice, half of the EPO-treated SJL/J EAE animals rapidly developed clinical symptoms related to erythrocytosis and expired after $<7$ days of EPO therapy (500-1000 U/kg/day). Peripheral blood from surviving mice showed a greatly elevated red cell mass (hematocrit $72-75 \%$ in whole EPO-treated mice $v s$ hematocrit $50-54 \%$ in controls; $p<0.05$ ). When lower doses of full-length EPO (50-500 U/kg) were serially tested for their hematopoietic effect in SJL/J mice, we found that doses $>75 \mathrm{U} / \mathrm{kg} /$ day for $5-7$ days significantly increased red cell mass, and doses $>250 \mathrm{U} / \mathrm{kg} /$ day for 7 days led to mortality rates of $30-50 \%$.

We compared the erythropoietic effect of our truncated synthetic EPO peptides to that of full-length EPO by measuring hematocrit changes in normal SJL/J mice after daily intravenous administration of whole EPO (100 U/kg) versus EPOderived short peptides $(250 \mu \mathrm{g} / \mathrm{kg})$ for 7 days. Normal SJL/J mice receiving PBS alone served as the untreated control group. As Fig. 1 shows, the hematocrit increased dramatically in SJL/J mice after receiving full-length EPO at $100 \mathrm{U} / \mathrm{kg}$ for 7 days and the hematocrit continued to rise to $\sim 75 \%$ by day 14 .

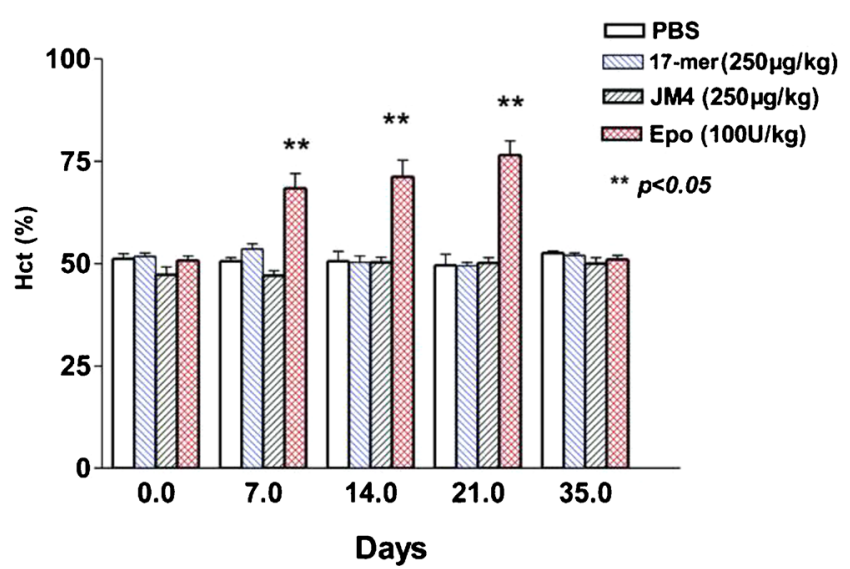

Fig. 1 Short erythropoietin (EPO)-derived peptides do not elevate hematocrit (Hct) levels in SJL/J mice. Mice were exposed to daily intravenous full-length EPO, short EPO-derived peptides (17-mer and JM-4), or phosphate-buffered saline (PBS) alone for 7 days $(n=5$ each group). Blood was collected for 5 weeks to assess changes in hematocrit. The hematocrit in full-length EPO $(100 \mathrm{U} / \mathrm{kg})$-treated SJL/J mice increased dramatically $(\sim 75 \%)$ within 1 week of EPO injection and remained elevated through to day 21 . In contrast, the hematocrit in the PBS sham-treated or short EPO peptides-treated group remained unaltered at $\sim 51 \pm 1 \%$
In contrast, hematocrit levels in groups treated with either PBS alone or EPO peptides (17-mer or JM-4) remained unchanged at $\sim 51 \pm 1 \%$ over the entire 5 -week period of follow-up. The hematocrit in animals treated with full-length EPO declined to normal values 4 weeks after whole-molecule EPO therapy ceased (day 35).

\section{EPO-derived Peptide JM-4 Exerts Strong Tissue Protective Effects and Reduces Clinical Disability in EAE}

The nonerythropoietic 17-mer linear peptide and the 19-mer JM-4 peptide were initially selected to determine their neuroprotective efficacy on acutely symptomatic EAE animals. However, we found that the 17-mer linear peptide induced minor and inconsistent clinical neurologic improvement in these EAE mice. In contrast, JM-4 peptide that contains the cysteine disulfide bond motif clearly possessed more consistent beneficial effects than the 17-mer linear peptide and all other EPO-derived short peptides from our peptide library. Thus, JM-4 was selected for all subsequent animal protection experiments. Figure 2a demonstrates that PLP antigen immunization induced significant neurologic disability by day 10 (clinical score 3-5). Symptomatic EAE mice were then either given intravenous JM-4 at $250 \mu \mathrm{g} / \mathrm{kg} /$ day in $200 \mu \mathrm{l}$ of PBS or sham-treated with PBS for 7 days. Early sustained clinical improvement occurred in the JM-4 treated group compared with the group receiving sham PBS treatment. Similar results were observed in experiments using the monophasic C57BL/6 EAE disease model. Significant clinical improvement was observed in both the EPO- and JM-4-treated groups compared with saline-treated EAE controls ( $p<0.03$; Fig. 2b). The JM-4 treated group showed only a trend toward more favorable clinical outcome compared with the EPO-treated group ( $p$ $=0.07$ ).

To determine if JM-4 could modify recurrent disease flair-ups and if symptom improvement could be sustained after termination of therapy, we employed a relapsingremitting model of EAE. We first immunized SJLJ mice with a suboptimal dose of PLP peptide antigen $(100 \mu \mathrm{g})$ on day 0 and allowed the mildly symptomatic PLPinduced EAE animals to recover without intervention. The recovered mice were subsequently divided into 2 groups that received further treatment with JM-4 or PBS for 7 days and were then challenged with a second immunization containing the same amount of PLP antigen. As Fig. 3 illustrates, previous JM-4 therapy delayed the onset of disease and signficantly reduced EAE-induced motor dysfunction compared with sham PBS-treated EAE mice $(p<0.05)$. More importantly, to determine if JM-4 possessed long-term therapeutic effects, we monitored animals for an additional 5 weeks without further pharmacological intervention. While the sham PBS-treated group continued to exhibit protracted relapses and more 


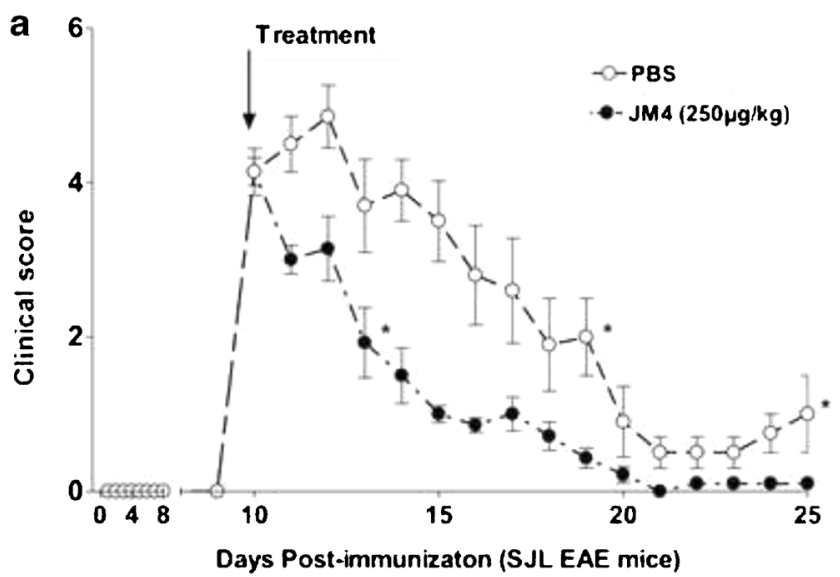

b

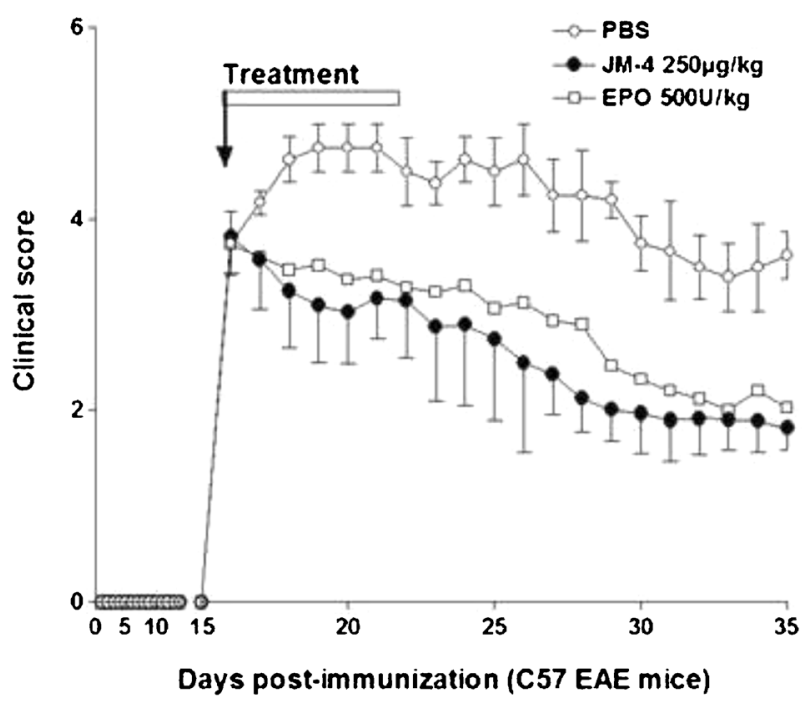

Fig. 2 JM-4 rapidly induces clinical recovery in acute proteolipid protein (PLP)-experimental autoimmune encephalomyelitis (EAE) and myelin oligodendrocyte glycoprotein (MOG) -EAE. (a) PLP immunized SJL/J mice ( $n=10$ per group) developed significant neurologic impairment at approximately 10 days postimmunization. When JM-4 was administered at the onset of symptoms for 7 days, clinical improvement appeared after 1 day, which was in contrast with EAE animals sham-treated with phosphate-buffered saline (PBS) $(p<0.005)$. This reduction in symptom severity was sustained even after cessation of treatment. The experiments were repeated 4 times with similar clinical outcome. (2) MOG-induced EAE in the C57BL/6 mouse showed a disease onset around day $15-16$ postimmunization. The symptomatic animals $(n=5-$ 7 /group) were divided randomly into 3 groups that received daily intravenous EPO (500 U/kg/day for 7 days) or JM-4 $(250 \mu \mathrm{g} / \mathrm{kg} /$ day for 7 days), or saline for the same period. Significant clinical improvement was observed in both EPO- and JM-4-treated groups when compared with saline-treated EAE controls $(p<0.03)$. The JM-4 treated group showed similar clinical outcome when compared with the EPO-treated group $(p=0.072)$

pronounced neurologic deficit, the JM-4-treated EAE mice manifested very mild disease with little or no hindlimb impairment $(p<0.001)$. JM-4 consistently mediated neuroprotective efficacy in each repeated experiment with stocks of peptide that had been stored under the same conditions for up to 6-12 months.

\section{JM-4 Treatment Protects Against Demyelination and Axonal Damage in the Acute EAE Spinal Cord}

We sought to determine whether the characteristic histopathological abnormalities found in EAE spinal cord at the peak of disease would be improved by JM-4 treatment. Luxol fast blue periodic acid-Schiff staining and SMI-32 labeling of nonphosphorylated neurofilament $\mathrm{H}$ was employed to assess myelin and axonal integrity, respectively, on spinal cord sections from EAE mice with or without JM-4 treatment. Figure 4 shows that JM-4 prevented demyelination, as depicted by blocking of white matter vacuolization and reduction in associated myelin loss when compared with PBStreated EAE mice. Sham-treated EAE animals exhibited intense staining of nonphosphorylated neurofilament $\mathrm{H}$ in damaged axons in white matter regions, reflecting widespread acute axonal injury. In contrast, JM-4-treated mice showed far fewer SMI-32-labeled injured axons. Blinded ranked pair analysis indicated the difference between JM-4-treated animals and sham-treated control groups was significant $(p<$ $0.05)$.

\section{JM-4 Treatment Modifies Inflammatory or Immune Cell Numbers in EAE Peripheral Lymphatics}

The DILNs from the JM-4 or whole EPO, or Sham-treated C57BL/6 EAE mice were obtained after 3-day or 10-day treatment to determine analogous immunomodulating effects within the peripheral lymphoid tissue of EAE mice. We found much higher total MNC numbers in sham-treated EAE mice compared with normal mice without EAE $(p<0.005)$. Both whole EPO and JM-4 therapy dramatically reduced the total number of MNCs and greatly reduced the population of CD11c+ DCs (Table 1). Similar to treatment experiments in C57BL/6 EAE mice, JM-4 treatment in SJL/L EAE mice induced significant expansion of the Treg cell population and reduced $\mathrm{T}$ helper $(\mathrm{Th}) 17$ cells within the spleen tissues (Fig. 5).

\section{JM-4 Suppresses Proinflammatory Cytokine Production in MOG Peptide Enriched T Cells}

Enriched MOG peptide-specific T cells generated from inguinal lymph nodes and spleen of MOG-induced EAE animals were subsequently treated in vitro for $72 \mathrm{~h}$ with JM-4 or a nonspecific control peptide. T-cell culture supernatants were collected for cytokine profile analysis [12]. Table 2 shows that a substantial reduction in major proinflammatory cytokine levels (IL-2, IL-6, tumor necrosis factor- $\alpha$ and interferon- $\gamma$ ) was induced in cells treated in vitro with JM-4. It should be noted that both the full-length EPO molecule and JM-4 had little effect on IL-5 secretion. An irrelevant control peptide also had no effect on cytokine production. 


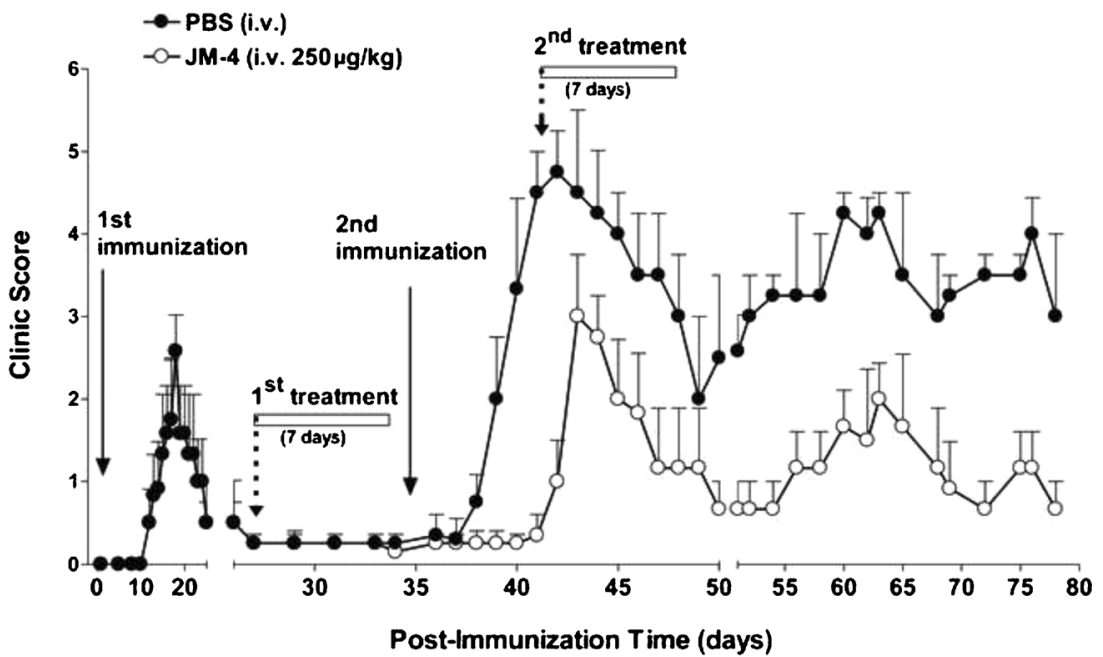

Fig. 3 JM-4 peptide treatment improves clinical course in relapsingremitting experimental autoimmune encephalomyelitis. SJL/J mice $(n=$ 6-10 per group) were immunized with a suboptimal dose of proteolipid protein (PLP) peptide $(100 \mu \mathrm{g})$ on day 0 , which produced only mild clinical deficits. Four weeks after the first immunization, all animals recovered and exhibited near normal clinical condition. These animals were then divided into 2 groups that received 7 days of treatment with either JM-4 peptide or sham phosphate-buffered saline (PBS) before a second PLP immunization was given. There was a delay in the onset of

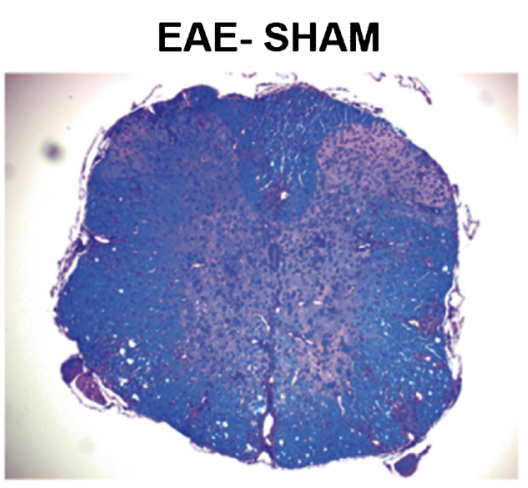

Luxol Fast Blue (Myelin)

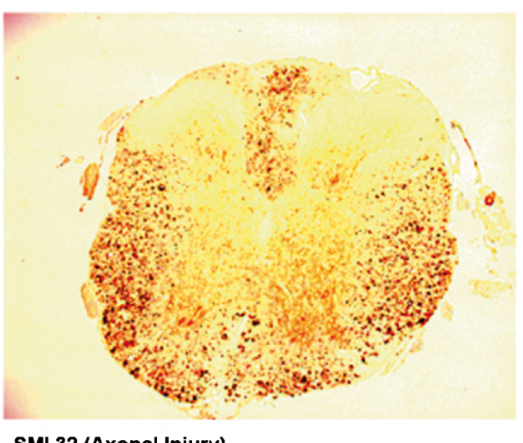

SMI 32 (Axonal Injury)

Fig. 4 JM-4 protects against spinal cord demyelination and axonal injury in experimental autoimmune encephalomyelitis (EAE). SJL/J mice immunized with proteolipid protein (PLP) peptide were treated with either JM-4 or phosphate-buffered saline for 7 days and spinal cords were collected to evaluate pathological differences by staining with Luxol fast blue for myelin, or labeled with SMI-32 antibody for nonphosphorylated neurofilament $\mathrm{H}$, a marker of acute axonal injury. In EAE sham-treated animals, vacuolated regions could be seen in the white

symptoms and reduction in severity in the JM-4-treated group compared with the sham-treated EAE animals $(p<0.005)$. A rapid reduction in clinical score occurred again when the JM-4-treated mice received a second course of JM-4 therapy. Long-term follow-up showed a relapsing and remitting pattern. However, the PBS-treated EAE mice showed far more severe disease, whereas EAE mice treated with JM-4 had much milder disease. The drug effect continued for the entire 40-day period after treatment was completed $(p<0.001)$. The repeat experiment showed similar clinical results

EAE - JM-4

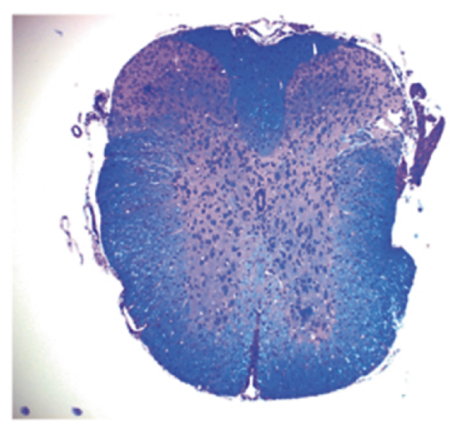

Luxol Fast Blue (Myelin)

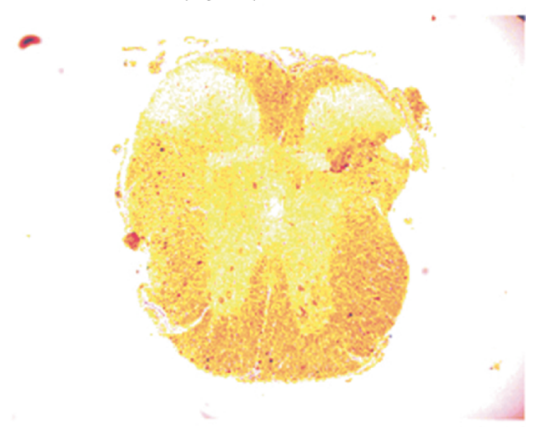

SMI 32 (Axonal Injury)

matter representing extensive demyelinated areas while JM-4 peptide treated EAE animals had little-to-no damage. Similarly, there was a pronounced reduction in number of injured SMI-32-stained axons in JM-4-treated EAE animals compared with sham-treated EAE controls. Blinded ranked pair analysis indicated the difference between JM-4treated animals ( $\mathrm{n}=5-6$ mice/group) and sham-treated control groups was significant $(p<0.05)$ 
Table 1 Effects of full erythropoietin and JM-4 peptide on total mononuclear cells (MNC), dendritic cells (DC), and regulatory $\mathrm{T}$ cells (Treg) in experimental autoimmune encephalomyelitis (EAE) lymph nodes

\begin{tabular}{llllll}
\hline & MNC $\left(\times 10^{5}\right)$ & Treg & & DC \\
\hline 3-Day treatment* & & $\times 10^{5}$ & $\%$ & $\times 10^{5}$ & $\%$ \\
Normal & $63.4 \pm 7.1$ & $2.35 \pm 0.08$ & $3.7 \pm 1.1$ & $0.110 \pm 0.001$ & $0.17 \pm 0.01$ \\
EAE: sham Rx & $204.1 \pm 21.3^{\dagger}$ & $4.28 \pm 0.20$ & $2.1 \pm 0.9$ & $3.880 \pm 0.002^{\dagger}$ & $1.90 \pm 0.10$ \\
EAE: full-length peptide & $92.3 \pm 10.2$ & $2.90 \pm 0.05$ & $3.1 \pm 0.5$ & $0.340 \pm 0.001$ & $0.37 \pm 0.03$ \\
EAE: JM-4 peptide & $94.2 \pm 13.1$ & $3.49 \pm 0.15$ & $3.7 \pm 1.1$ & $0.200 \pm 0.005$ & $0.21 \pm 0.04$ \\
10-Day treatment & & & & & \\
Normal & $72.1 \pm 9.9$ & $2.24 \pm 0.02$ & $3.1 \pm 0.2$ & $0.1400 \pm 0.0001$ & $0.20 \pm 0.01$ \\
EAE: sham Rx & $252.4 \pm 34.3^{\dagger}$ & $2.42 \pm 0.05^{\dagger}$ & $0.96 \pm 0.15$ & $4.79 \pm 0.08^{\dagger}$ & $1.9 \pm 0.2$ \\
EAE: full-length peptide & $82.0 \pm 21.2$ & $6.89 \pm 0.32^{\S}$ & $8.4 \pm 1.5$ & $0.180 \pm 0.002$ & $0.22 \pm 0.01$ \\
EAE: JM-4 peptide & $61.1 \pm 13.4$ & $5.56 \pm 0.24^{\S}$ & $9.1 \pm 1.8$ & $0.070 \pm 0.001$ & $0.11 \pm 0.01$ \\
\hline
\end{tabular}

Data are represented as mean \pm SEM

* $\mathrm{C} 57 \mathrm{BL} / 6$ mice were immunized with myelin oligodendrocyte glycoprotein peptide, and received treatment with sham phosphate-buffered saline (PBS), or EPO, or JM-4 daily. Animals ( $n=3$ each group) were sacrificed 3 days post-treatment with PBS, or EPO or JM-4 peptide, and inguinal lymph nodes were harvested for designed experiments

${ }^{\dagger} p<0.005$ compared with normal control, EPO-treated, or JM-4 treated mice

* $\mathrm{C} 57 \mathrm{BL} / 6$ mice were immunized with myelin oligodendrocyte glycoprotein peptide peptide, and received treatment with sham PBS, or EPO, or JM-4 daily. Animals ( $n=3$ each group) were sacrificed 10 days posttreatment with PBS, or EPO or JM-4 peptide, and inguinal lymph nodes were harvested for designed experiments ${ }^{\S} p<0.001$ compared with PBS sham-treated EAE mice

\section{JM-4 Provides Neuroprotection Against Cytotoxic Insult}

Rat neuroendocrine cell line PC12 was differentiated with nerve growth factor and then pretreated with either JM-4 or a scrambled inactive version of JM-4. The cells were exposed to neurotoxic $A \beta_{25-35}$ peptide for $24 \mathrm{~h}$ and subsequently harvested and analyzed for cell death following staining with 7$\mathrm{AAD}$ or annexin $\mathrm{V}$ for apoptosis [26]. Flow cytometry indicated that toxic $A \beta$ exposure induced a massive increase in apoptotic neural crest-derived cells that was fully blocked by pretreatment with JM-4, whereas scrambled JM-4 failed to protect cells from apoptosis (Fig. 6).

\section{Neuroprotection Against Cell Death is Limited to an Early Segment of the Whole EPO Molecule}

Cultured rat $\mathrm{PC} 12$ cells were differentiated and then pretreated with JM-4, scrambled JM-4, EPO, or 1 of 6 peptide segments derived from EPO (labeled EP-P\#1-EP-P\#6; see "Materials and Methods" for details) at a concentration of $1 \mu \mathrm{g} / \mathrm{ml} 2 \mathrm{~h}$ prior to adding $A \beta_{25-35}$ peptide. Twenty-four hours after the addition of $\mathrm{A} \beta_{25-35}$ peptide, cells were analyzed by fluorescence-activated cell sorting. Control cells grown in media without toxic exposure exhibited baseline rates of apoptosis below $5-10 \%$ (data not shown). A high proportion of apoptosis was observed in PC12 cells that were exposed to toxic $\mathrm{A} \beta$ without pretreatment while much reduced levels of apoptosis were observed in cells pretreated with either whole EPO or JM-4 peptide (Fig. 7). Among the segments of EPO that were tested for cytoprotective effectiveness, EP-P\#2 (amino acid T26-50W) was the only one to demonstrate marked reduction in proportion of apoptotic cells following toxic exposure to $A \beta$. Scrambled sequence JM-4 failed to reduce apoptosis in this assay.

\section{Discussion}

Tissue protective effects of full-length EPO in preclinical studies have been widely reported for a variety of CNS pathologies in recent years [1, 2, 6, 8-10, 27, 28], but a major obstacle to using whole EPO as a long-term therapeutic is the induction of potentially hazardous increases in red cell mass and overall mortality $[15,27]$. To address this problem, we successfully designed truncated small linear or cyclic EPO-derived peptides from various domains of the full-length molecule and selected the best one for animal models of EAE. Our studies with JM-4 peptide demonstrated marked beneficial effects on acute neurologic symptoms in both MOG and PLP peptideinduced EAE that was unaccompanied by hematologic or other side effects (Figs. 2 and 3).

A beneficial effect of full-length EPO treatment in C57BL/ 6 MOG-EAE mice has been demonstrated by our group and others [7, 29]. Long-term full-length EPO treatment, even at relatively high doses, failed to induce significant erythropoietic side effects in C57BL/6 mice. However, when analogous experiments were conducted in PLP-induced SJL/L EAE mice, EPO therapy greatly increased the hematocrit and SJL/ 
a

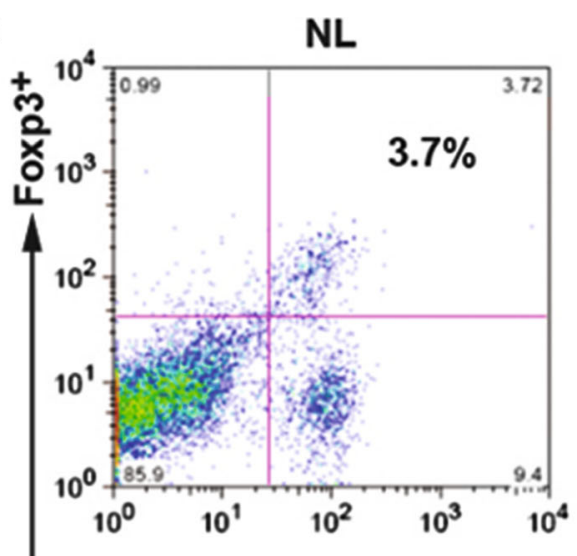

PBS

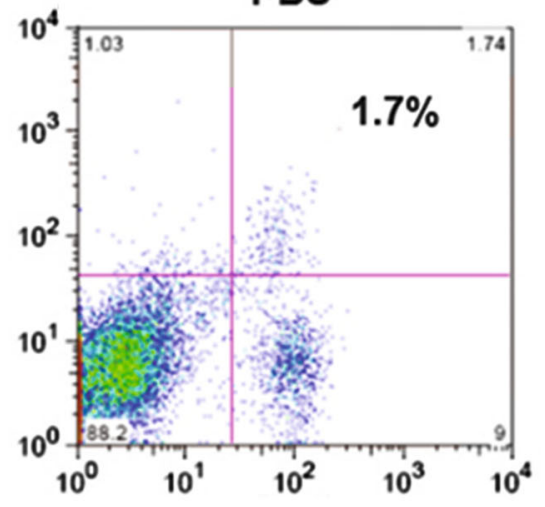

JM-4

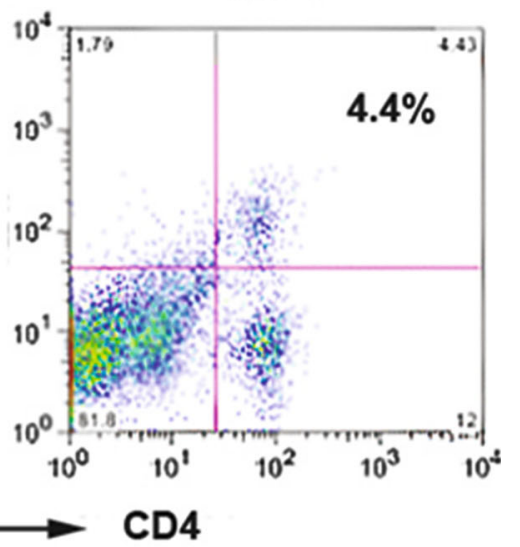

b
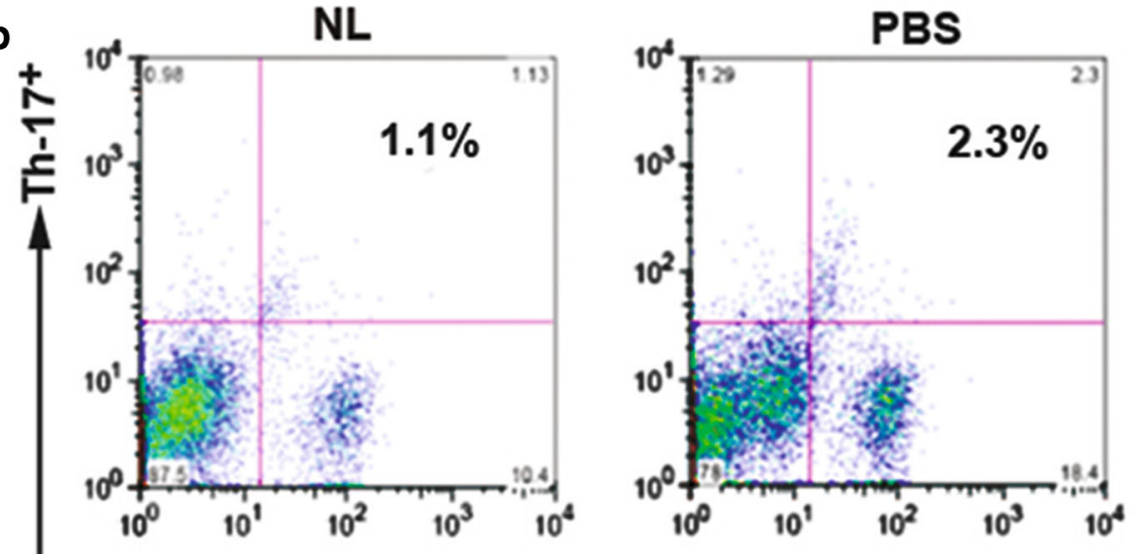

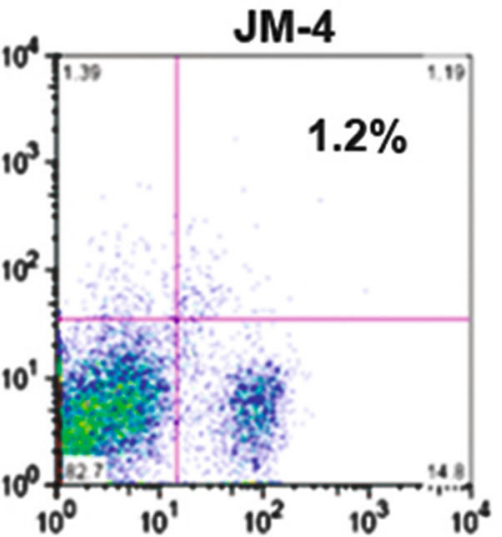

CD4
Fig. 5 JM-4 peptide induced expansion of regulatory T (Treg) cells and reduced T helper (Th)17-positive cells. SJL/J mice immunized with proteolipid protein (PLP) peptide were treated with either JM-4 or phosphate-buffered saline (PBS) for 7 days. Animals $(n=3$ per treatment group) were sacrificed after 7 days treatment with PBS or JM-4 peptide and spleens were harvested for designed experiments. (Top panel) A significantly reduced number of splenic Treg cells was detected in sham-treated experimental autoimmune encephalomyelitis

$\mathrm{J}$ mice were unable to tolerate regular doses of full-length EPO. There is, to our knowledge, only 1 report thus far using
(EAE) control mice when compared with normal SJL/J controls $(1.7 \%$ vs $3.7 \%$; $p<0.001$ ), whereas JM-4 treatment for 7 days sharply increased cell splenic Foxp3 numbers $(6.9 \%$ vs $1.7 \% ; p<0.001)$. (Bottom panel) Increased splenic Th17 cells were detected in sham-treated EAE control mice $(2.3 \% v s 1.2 \% ; p<0.005)$. JM-4 therapy for 7 days in EAE mice significantly reduced number of Th17 cells to normal levels $(1.1 \% \mathrm{vs}$ $1.2 \%) . N L=$ Normal

high-dose full-length EPO to treat SJL/J EAE mice [30]. In contrast to the report by Zhang et al. [30], our experiments

Table 2 Effects of erythropoietin (EPO) and its derived small peptide JM-4 suppress enriched experimental autoimmune encephalomyelitis (EAE) T cell cytokine secretion

\begin{tabular}{llllll}
\hline & IL-2 & IL-5 & IL-6 & TNF- $\alpha$ & IFN- $\gamma$ \\
\hline No treatment & $544.7 \pm 42.2$ & $219.3 \pm 37.4$ & $424.7 \pm 21.9$ & $330.1 \pm 36.4$ & $4020.3 \pm 156.1$ \\
Control peptide $(1 \mathrm{ng} / \mathrm{ml})$ & $552.1 \pm 37.6$ & $224.0 \pm 44.0$ & $350.7 \pm 16.3$ & $310.6 \pm 44.7$ & $4000.1 \pm 171.2$ \\
Full-length EPO $(1 \mathrm{U} / \mathrm{ml})$ & $336.2 \pm 41.7^{*}$ & $216.2 \pm 39.3$ & $240.1 \pm 42.8^{*}$ & $200.4 \pm 41.7^{*}$ & $2600.7 \pm 188.3^{*}$ \\
JM-4 $(1 \mathrm{ng} / \mathrm{ml})$ & $319.9 \pm 12.7^{*}$ & $192.7 \pm 22.2$ & $248.0 \pm 31.3^{*}$ & $160.9 \pm 11.8^{\dagger}$ & $2160.0 \pm 104.5^{\dagger}$ \\
\hline
\end{tabular}

T cells were collected and purified from spleen and draining inguinal lymph nodes of C57BL/6 EAE mice ( $\mathrm{n}=3-5$ mice/group) 5-7 days after the second immunization with myelin oligodendrocyte glycoprotein $_{35-55}$

Data are represented as mean \pm SEM, performed in triplicate

$* p<0.05$ compared with no treatment or control peptide treatment

${ }^{\dagger} p<0.01$ compared with no treatment or control peptide treatment 

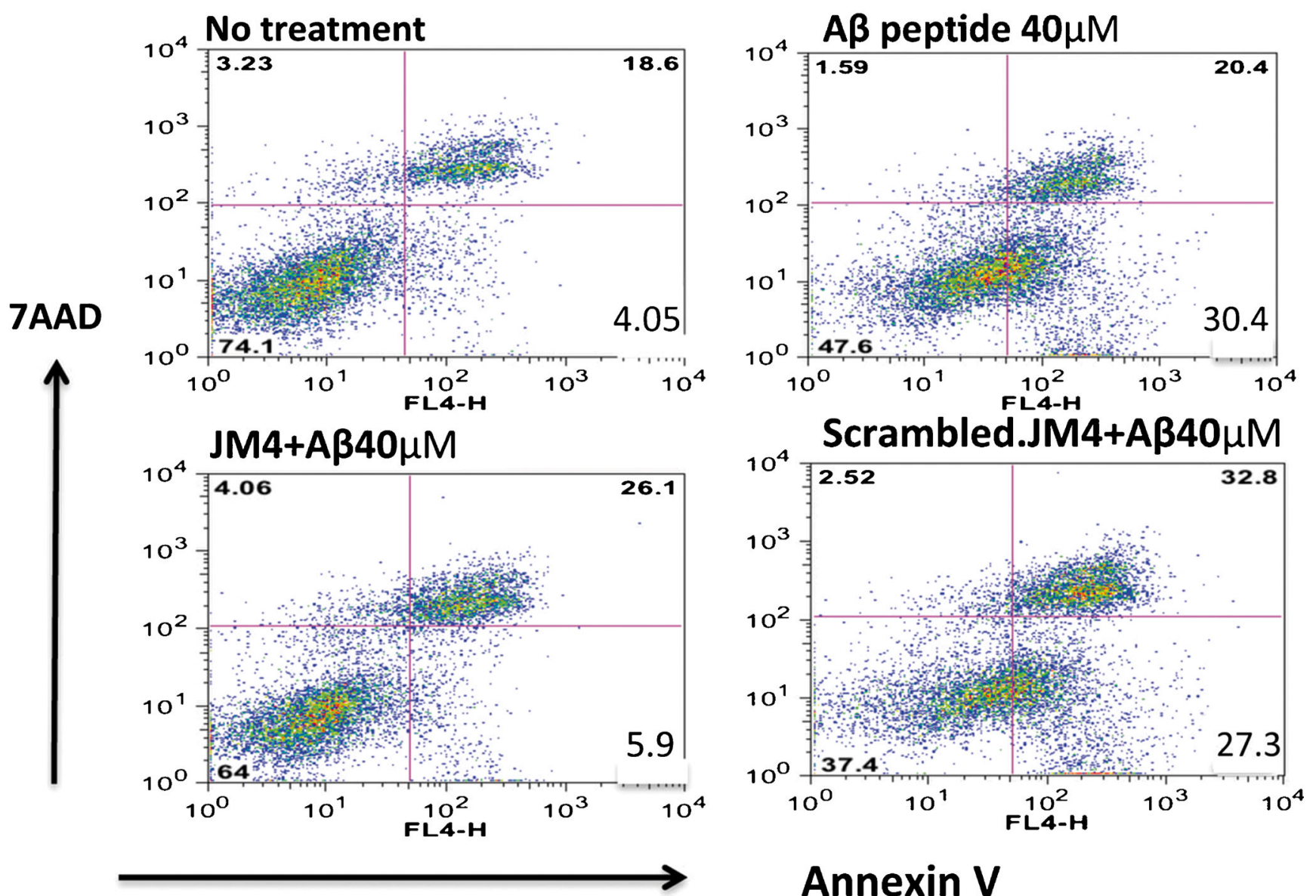

Annexin V

Fig. 6 JM-4 attenuates cytotoxin-induced neuronal apoptosis. The neural crest-derived rat pheochromocytoma cell line PC12 was differentiated in vitro for $24 \mathrm{~h}$. We pretreated cultures with JM-4 or scrambled JM-4 at a concentration of $1 \mu \mathrm{g} / \mathrm{ml}$ for $2 \mathrm{~h}$, and then challenged the cells with $\mathrm{A} \beta_{25-35}$ peptide at $40 \mu \mathrm{M}$ for $24 \mathrm{~h}$. The cells were harvested and analyzed for death using 7-amino-actinomycin D (7-AAD), and stained for apoptosis with allophycocyanin conjugated-annexin V. The washed reacted cells were analyzed by flow cytometry. The lower right quadrant

repeatedly showed that neither normal SJL/J nor SJL/J EAE mice were capable of tolerating high-dose full-length EPO therapy. More than $50 \%$ of treated SJL/J mice died of complications from overwhelming erythropoiesis after 5-7 days of high-dose EPO (1000-5000 U/kg/day) treatment. We believe the different erythropoietic response to the whole EPO molecule by C57BL/6 versus $\mathrm{SJL} / \mathrm{J}$ mouse strains may be caused by different genetic elements. Our findings may also explain, in part, the paucity of full-length EPO molecule studies reported in the commonly used SJL/J EAE mouse model.

Structure-function studies on EPO have suggested that 2 distinct activities (erythropoiesis and tissue protection) reside in different domains within the intact EPO molecule [2, 31, 32]. Several approaches have been used to modify full-length EPO in order to eliminate the erythropoietic effects while retaining the desired tissue-protective effects [2, 33-35]. Desialylated EPO and carbamylated EPO are commonly modified forms of EPO used today for tissue protection $[35,36]$. values within each graph show the size of the annexin V-positive apoptotic cell population. Toxic $\mathrm{A} \beta$ peptide exposure induced a massive increase in apoptotic cells (30.4\%), whereas exposure to a reverse sequence $A \beta_{35-25}$ molecule failed to induce apoptosis (data not shown). In contrast, JM-4 treatment strongly blocked apoptosis (reduced to $5.9 \%$ ), whereas therapy with scrambled JM-4 failed to protect cells from apoptosis

Preclinical animal studies have shown that these modified EPO molecules still retain their neuroprotective effects in MOG-induced EAE, as well as in stroke and traumatic brain injury $[6,36,37]$.

Cyclic peptide structures occur naturally within proteins and are known to play important roles in peptide stability, which is critical for maintaining their consistent biologic effects. Several small EPO-derived peptides from our peptide library were found to retain their inclusive tissue-protective effects seen with full-length EPO without producing hematocrit alterations in SJL-J EAE mice. Among these small EPO peptides, the 19-mer JM-4 peptide derived from the EPO-AB loop with 2 cysteine molecules, demonstrated more profound and consistent beneficial effects than those with a single cysteine in their structure. A 17-mer peptide derived from the EPO-AB loop that contains 1 cysteine molecule was once reported to have neuroprotective/trophic effects in cell culture, though little subsequent in vivo data appeared showing 
Fig. 7 Neuroprotection against cell death is limited to an early segment of the whole erythropoietin (EPO) molecule. $\mathrm{PC} 12$ cells were exposed in vitro to toxic $\mathrm{A} \beta_{25-35}$ peptide to induce cell death. The apoptotic cell fraction from fluorescenceactivated cell sorting analysis was calculated to quantify the proportion of dead and dying cells. Toxic exposure to $A \beta$ peptide induced large levels of cellular apoptosis that was dramatically reduced by pretreatment with whole EPO, JM-4 peptide, or the second segment of EPO (EP-P\#2) ${ }^{* *} p<0.01,{ }^{* * *} p<0.001$

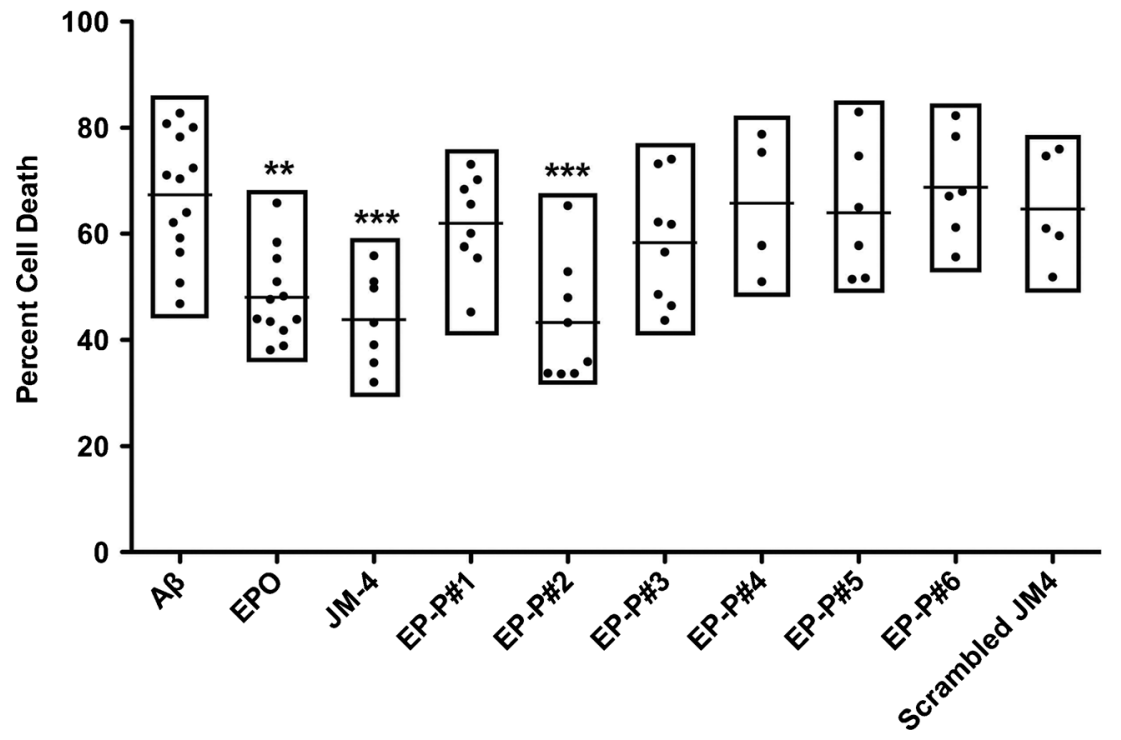

Treatment efficacy in neurologic or tissue protection [38]. We observed clinical improvement once in SJL/J EAE mice with freshly prepared 17-mer peptide, but all subsequent experiments with the 17-mer peptide failed to confirm its clinical benefit in SJL/ $\mathrm{J}$ or C57BL/6 EAE mice. The inconsistency of the 17-mer peptide-mediated effect in EAE animal models prompted us to analyze the 17-mer by high-performance liquid chromatography combined with mass spectroscopy for stability. We found that the 17-mer linear peptide easily disintegrated into small pieces, especially after being stored in a freezer for $>7$ 10 days (data not shown). This finding may explain the initial lack of enthusiasm in the research on EPO-derived small peptides over the last decade since O'Brien et al. [38] identified the original 17 amino acid linear EPO-derived peptide.

To this end we have determined that the zone containing the bulk of the tissue protective effect is located in the early third of the molecule within the A $\beta$ loop of EPO. JM- 4 markedly altered the course of EAE in both C57BL/6 and SJL/J mice and attenuated the severity of clinical disease in both acute relapsing-remitting and monophasic forms of the disease without inducing erythropoiesis. In the acute model of EAE, JM-4 reduced the clinical score of EAE at symptom onset and this effect persisted long term, even after treatment ceased. JM-4 delayed onset, reduced disability at the peak of disease, and attenuated relapses when animals were challenged with the same PLP antigen. It is important to demonstrate therapeutic effect during early neurologic presentation because it more accurately reflects the human clinical situation. Therapies that are effective at symptom onset and provoke long-term resolution of subsequent relapses would be desirable for MS treatment. In the preclinical animal model data presented here, our agents have performed satisfactorily in this regard.
Additionally, we demonstrated that JM-4 blocked apoptosis when neurons were exposed to a neurotoxic environment, shedding light on 1 of several possible mechanisms by which the motif provides neuroprotection. Cytotoxicity assays demonstrated that the neuroprotective effect of EPO is, in large part, within a single segment of the full-length molecule, the same segment (T26-50W) that contains the JM-4 peptide sequence. A similar experiment measuring neural cell death using the dUTP nick end labeling assay in an animal model of traumatic brain injury also demonstrated that most of the neuroprotective effects of EPO are housed in the early portion of the molecule (unpublished data). Taken together, these results strongly suggest that the effectiveness of JM-4 peptide toward neuroprotection takes advantage of a specific early region within the whole EPO molecule.

The disease process in EAE leads to a characteristic CNS inflammatory infiltrate that contributes to the damage observed in the spinal cord and brain. $T$ cells and other immune effectors are believed to be important in the pathogenesis of EAE [39-50]. In the EAE animal models, JM-4 demonstrates a remarkable capacity to significantly reduce DC, Th17 cell numbers, block T-cell proliferation and proinflammatory cytokine production, as well as reduce demyelination and axonal injury. Our studies demonstrated JM-4 significantly reduced mononuclear, Th17, and DC numbers in lymphoid tissue with concomitant expansion of Treg cells that is sustained long after the treatment period. DCs are important in the induction and priming stages of EAE, and thereby orchestrate the events that lead to $\mathrm{CD} 4+/ \mathrm{Th} 17$ polarization and the development of autoimmunity [51, 52]. Interestingly, previous studies conducted in our laboratory have shown that whole EPO molecule clearly shifts the balance away from a Th17 polarizing response [12]. Studies have reported that DC precursors 
accumulate in the blood before relapses and that selective ablation of these cells reduces disease severity [53]. It is attractive to hypothesize that the DC effector response can be viewed as a novel upstream therapeutic target for selective modulation of the innate immune system. We are currently involved in studies to define the role of these cells as key players in mediating the curative effects of our EPO-derived peptides.

In conclusion, our studies demonstrate that a significant beneficial effect of EPO-derived short peptide JM-4 therapy on the acute neurologic deficit in EAE can be readily achieved without encountering any hematologic side effects. It may hold considerable potential for direct clinical application in the treatment of neuroinflammatory diseases, demyelinating illnesses, CNS trauma, and stroke, as well as in the treatment of inflammatory/immune diseases of non-neural origins.

Acknowledgments This work was supported by grants from the Veterans Affairs Office of Research and Development (IO1 RX000220-01; PD and RX001305-01A2; PD), the Segal Fund, and a National Institutes of Health Bridging Interventional Development Gaps (formerly National Institutes of Health RAID) award (1 X01 NS0073526-01A1; PD). Full conflict of interest disclosure is available in the electronic supplementary material for this article.

Required Author Forms Disclosure forms provided by the authors are available with the online version of this article.

\section{References}

1. Brines ML, Ghezzi P, Keenan S, et al. Erythropoietin crosses the blood-brain barrier to protect against experimental brain injury. Proc Natl Acad Sci U S A 2000:97;10526-10531.

2. Brines M, Grasso G, Fiordaliso F, et al. Erythropoietin mediates tissue protection through an erythropoietin and common betasubunit heteroreceptor. Proc Natl Acad Sci U S A 2004: 101;14907-14912.

3. Buemi M, Cavallaro E, Floccari F, et al. Erythropoietin and the brain: from neurodevelopment to neuroprotection. Clin Sci 2002: 103;275-282.

4. Siren AL, Ehrenreich H. Erythropoietin - a novel concept for neuroprotection. Eur Arch Psychiatry Clin Neurosci 2001:251;179184.

5. Buemi M, Cavallaro E, Floccari F, et al. The pleiotropic effects of erythropoietin in the central nervous system. J Neuropathol Exp Neurol 2003:62;228-236.

6. Wang Y, Zhang ZG, Rhodes K, et al. Post-ischemic treatment with erythropoietin or carbamylated erythropoietin reduces infarction and improves neurological outcome in a rat model of focal cerebral ischemia. Br J Pharmacol 2007:151;1377-1384.

7. Li W, Maeda Y, Yuan RR, et al. Beneficial effect of erythropoietin on experimental allergic encephalomyelitis. Ann Neurol 2004: $56 ; 767-777$.

8. Grasso G, Graziano F, Sfacteria A, et al. Neuroprotective effect of erythropoietin and darbepoetin alfa after experimental intracerebral hemorrhage. Neurosurgery 2009:65;763-769.

9. Meng Y, Xiong Y, Mahmood A, et al. Dose-dependent neurorestorative effects of delayed treatment of traumatic brain injury with recombinant human erythropoietin in rats. $\mathrm{J}$ Neurosurg 2011:115;550-560.

10. Sakanaka M, Wen TC, Matsuda S, et al. In vivo evidence that erythropoietin protects neurons from ischemic damage. Proc Natl Acad Sci U S A 1998:95;4635-4640.

11. Tsai PT, Ohab JJ, Kertesz N, et al. A critical role of erythropoietin receptor in neurogenesis and post-stroke recovery. J Neurosci 2006: 26;1269-1274.

12. Yuan RR, Maeda Y, Li WP, et al. Erythropoietin: a potent inducer of peripheral immuno/inflammatory modulation in autoimmune EAE. PLoS One 2008:3.

13. Dicato M. Venous thromboembolic events and erythropoiesisstimulating agents: An update. Oncologist 2008:13;11-15.

14. Corwin HL, Gettinger A, Fabian TC, et al. Efficacy and safety of epoetin alfa in critically ill patients. N Engl J Med 2007:357;965976.

15. Ehrenreich $\mathrm{H}$, Weissenborn $\mathrm{K}$, Prange $\mathrm{H}$, et al. Recombinant human erythropoietin in the treatment of acute ischemic stroke. Stroke 2009:40;E647-E656.

16. Yoshimura T, Kunishita T, Sakai K, et al. Chronic experimental allergic encephalomyelitis in guinea pigs induced by proteolipid protein. J Neurol Sci 1985:69;47-58

17. Sobel RA, van der Veen RC, Lees MB. The immunopathology of chronic experimental allergic encephalomyelitis induced in rabbits with bovine proteolipid protein. J Immunol 1986:136;157-163.

18. Tuohy VK, Lu Z, Sobel RA, Laursen RA, Lees MB. Identification of an encephalitogenic determinant of myelin proteolipid protein for SJL mice. J Immunol 1989:142;1523-1527.

19. Greer JM, Kuchroo VK, Sobel RA, Lees MB. Identification and characterization of a second encephalitogenic determinant of myelin proteolipid protein (residues 178-191) for SJL mice. J Immunol 1992:149;783-788.

20. Trapp BD, Bo L, Mork S, Chang A. Pathogenesis of tissue injury in MS lesions. J Neuroimmunol 1999:98;49-56.

21. Bettelli E, Pagany M, Weiner HL, et al. Myelin oligodendrocyte glycoprotein-specific T cell receptor transgenic mice develop spontaneous autoimmune optic neuritis. J Exp Med 2003:197;10731081.

22. Crawford MP, Yan SX, Ortega SB, et al. High prevalence of autoreactive, neuroantigen-specific CD8+ T cells in multiple sclerosis revealed by novel flow cytometric assay. Blood 2004: $103 ; 4222-4231$

23. Abdul-Majid KB, Wefer J, Stadelmann C, et al. Comparing the pathogenesis of experimental autoimmune encephalomyelitis in CD4-/- and CD8-/- DBA/1 mice defines qualitative roles of different T cell subsets. J Neuroimmunol 2003:141;10-19.

24. Battistini L, Piccio L, Rossi B, et al. CD8+ T cells from patients with acute multiple sclerosis display selective increase of adhesiveness in brain venules: a critical role for P-selectin glycoprotein ligand-1. Blood 2003:101;4775-4782.

25. Bebo BF, Jr., Fyfe-Johnson A, Adlard K, et al. Low-dose estrogen therapy ameliorates experimental autoimmune encephalomyelitis in two different inbred mouse strains. J Immunol 2001:166;20802089.

26. Shanmugam G, Polavarapu PL. Structure of A beta(25-35) peptide in different environments. Biophys J 2004:87;622-630.

27. Bohlius J, Schmidlin K, Brillant C, et al. Erythropoietin or Darbepoetin for patients with cancer-meta-analysis based on individual patient data. Cochrane Database of Syst Rev 2009;CD007303.

28. Xiong Y, Chopp M, Lee CP. Erythropoietin improves brain mitochondrial function in rats after traumatic brain injury. Neurol Res 2009:31;496-502.

29. Chen SJ, Wang YL, Lo WT, et al. Erythropoietin enhances endogenous haem oxygenase-1 and represses immune responses to 
ameliorate experimental autoimmune encephalomyelitis. Clin Exp Immunol 2010:162;210-223.

30. Zhang J, Li Y, Cui Y, et al. Erythropoietin treatment improves neurological functional recovery in EAE mice. Brain Res 2005: 1034;34-39.

31. Wen D, Boissel JP, Showers M, Ruch BC, Bunn HF. Erythropoietin structure-function relationships. Identification of functionally important domains. J Biol Chem 1994:269;22839-22846.

32. Ahmet I, Tae HJ, Juhaszova M, et al. A small nonerythropoietic helix B surface peptide based upon erythropoietin structure is cardioprotective against ischemic myocardial damage. Mol Med 2011:17;194-200.

33. Erbayraktar S, Grasso G, Sfacteria A, et al. Asialoerythropoietin is a nonerythropoietic cytokine with broad neuroprotective activity in vivo. Proc Natl Acad Sci U S A 2003:100;6741-6746.

34. Leist M, Ghezzi P, Grasso G, et al. Derivatives of erythropoietin that are tissue protective but not erythropoietic. Science 2004:305;239242.

35. Mun KC, Golper TA. Impaired biological activity of erythropoietin by cyanate carbamylation. Blood Purif 2000:18;13-17.

36. Adembri C, Massagrande A, Tani A, et al. Carbamylated erythropoietin is neuroprotective in an experimental model of traumatic brain injury. Crit Care Med 2008:36;975-978.

37. Savino C, Pedotti R, Baggi F, et al. Delayed administration of erythropoietin and its non-erythropoietic derivatives ameliorates chronic murine autoimmune encephalomyelitis. J Neuroimmunol 2006:172;27-37.

38. Campana WM, Misasi R, O'Brien JS. Identification of a neurotrophic sequence in erythropoietin. Int J Mol Med 1998:1;235-241.

39. Huseby ES, Liggitt D, Brabb T, et al. A pathogenic role for myelinspecific CD8(+) T cells in a model for multiple sclerosis. J Exp Med 2001:194;669-676.

40. Sun D, Whitaker JN, Huang Z, et al. Myelin antigen-specific CD8+ T cells are encephalitogenic and produce severe disease in C57BL/6 mice. J Immunol 2001:166;7579-7587.

41. Sun D, Zhang Y, Wei B, et al. Encephalitogenic activity of truncated myelin oligodendrocyte glycoprotein (MOG) peptides and their recognition by CD8+ MOG-specific T cells on oligomeric MHC class I molecules. Int Immunol 2003:15;261-268.
42. Youssef S, Stuve O, Patarroyo JC, et al. The HMG-CoA reductase inhibitor, atorvastatin, promotes a Th2 bias and reverses paralysis in central nervous system autoimmune disease. Nature 2002:420;7884.

43. Chou YK, Culbertson N, Rich C, et al. T-cell hybridoma specific for myelin oligodendrocyte glycoprotein-35-55 peptide produced from HLA-DRB1*1501-transgenic mice. J Neurosci Res 2004:77;670680.

44. Montero E, Nussbaum G, Kaye JF, et al. Regulation of experimental autoimmune encephalomyelitis by CD4+, CD25+ and CD8+ T cells: analysis using depleting antibodies. J Autoimmun 2004:23;1-7.

45. Trajkovic V, Vuckovic O, Stosic-Grujicic S, et al. Astrocyteinduced regulatory $\mathrm{T}$ cells mitigate CNS autoimmunity. Glia 2004:47;168-179.

46. Faunce DE, Terajewicz A, Stein-Streilein J. Cutting edge: in vitrogenerated tolerogenic APC induce CD8+ T regulatory cells that can suppress ongoing experimental autoimmune encephalomyelitis. J Immunol 2004:172;1991-1995.

47. Dandekar AA, Anghelina D, Perlman S. Bystander CD8 T-cellmediated demyelination is interferon-gamma-dependent in a coronavirus model of multiple sclerosis. Am J Pathol 2004:164;363369.

48. Linker RA, Rott E, Hofstetter HH, et al. EAE in beta-2 microglobulin-deficient mice: axonal damage is not dependent on MHC-I restricted immune responses. Neurobiol Dis 2005:19;218-228.

49. Ford ML, Evavold BD. Specificity, magnitude, and kinetics of MOG-specific CD8+ T cell responses during experimental autoimmune encephalomyelitis. Eur J Immunol 2005:35;76-85.

50. Fontoura P, Garren H, Steinman L. Antigen-specific therapies in multiple sclerosis: going beyond proteins and peptides. Int Rev Immunol 2005:24;415-446.

51. Davidson MG, Alonso MN, Yuan R, et al. Th17 cells induce Th1polarizing monocyte-derived dendritic cells. J Immunol 2013: $191 ; 1175-1187$

52. Isaksson M, Ardesjo B, Ronnblom L, et al. Plasmacytoid DC promote priming of autoimmune Th17 cells and EAE. Eur J Immunol 2009:39;2925-2935.

53. Kang Z, Altuntas CZ, Gulen MF, et al. Astrocyte-restricted ablation of interleukin-17-induced Act1-mediated signaling ameliorates autoimmune encephalomyelitis. Immunity 2010:32;414-425. 